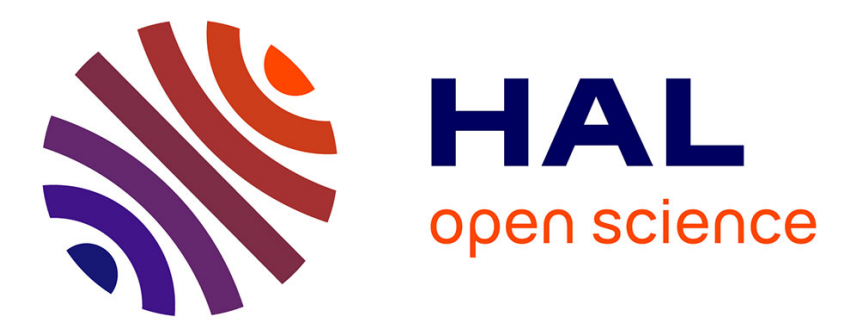

\title{
Dynamic inflation of hyperelastic spherical membranes
}

Erwan Verron, Roger Khayat, Abdelouahed Derdouri, Bernard Peseux

\section{To cite this version:}

Erwan Verron, Roger Khayat, Abdelouahed Derdouri, Bernard Peseux. Dynamic inflation of hyperelastic spherical membranes. Journal of Rheology, 1999, 43 (5), pp.1083-1097. 10.1122/1.551017. hal-01006758

\section{HAL Id: hal-01006758 https://hal.science/hal-01006758}

Submitted on 16 Jun 2014

HAL is a multi-disciplinary open access archive for the deposit and dissemination of scientific research documents, whether they are published or not. The documents may come from teaching and research institutions in France or abroad, or from public or private research centers.
L'archive ouverte pluridisciplinaire $\mathbf{H A L}$, est destinée au dépôt et à la diffusion de documents scientifiques de niveau recherche, publiés ou non, émanant des établissements d'enseignement et de recherche français ou étrangers, des laboratoires publics ou privés.

\section{(c)(1)}

Distributed under a Creative Commons Attribution| 4.0 International License 


\title{
Dynamic inflation of hyperelastic spherical membranes
}

\author{
E. Verron \\ Laboratoire de Mécanique et Matériaux, Division Structures, Ecole Centrale de \\ Nantes, B.P. 92101, 44321 Nantes Cedex 3, France \\ R. E. Khayat
}

Department of Mechanical and Materials Engineering, The University of Western Ontario, London, Ontario, Canada N6A 5B9

A. Derdouri

Industrial Materials Institute, National Research Council of Canada, 75 De Mortagne Boulevard, Boucherville, Québec, Canada J 4B 6Y4

B. Peseux

Laboratorie de Mécanique et Matériaux, Division Structures, Ecole Centrale de Nantes, B.P. 92101, 44321 Nantes Cedex 3, France

The dynamic inflation of hyperelastic spherical membranes of a Mooney-Rivlin material is analyzed in this study. Various inflation regimes are identified through ranges of the material parameters and driving pressure. In particular, the conditions for oscillatory inflation around the static fixed point are examined. It is found that, for a given material, the frequency of oscillation exhibits a maximum at some pressure level, which tends to increase for materials closer to neo-Hookean behavior.

\section{INTRODUCTION}

While the stability of statically inflated nonlinear materials is well understood, that taking place during dynamic inflation is relatively unexplored. The existence of instabilities in statically inflated hyperelastic membranes is well known, and such instabilities were observed experimentally by Hart-Smith (1966) and Alexander (1971). The general equations for static inflation of a thin spherical shell were reviewed by Beatty (1987), and the stability of the static solution was extensively analyzed recently by Shang (1991) using bifurcation theory.

More recently, there has also been considerable interest in the dynamic inflation, taking into account the inertia of the membrane. Such investigations are reviewed in detail by Jenkins and Leonard (1991) and Jenkins (1996). The theoretical analysis of 
Akkas (1978) shows the existence of instabilities during the dynamic inflation of spherical membranes. His theory, however, assumes that the membrane thickness remains constant during inflation.

In this paper, we first review the governing equations for the motion of a spherical membrane for a material obeying the Mooney-Rivlin constitutive equation, subjected to an arbitrary and time-dependent driving pressure. A summary of the various regimes in static inflation is then given. The dynamic inflation is extensively investigated to generate both the linear stability picture and the nonlinear behavior. The influence of various control parameters, namely, the material constant(s) and driving pressure are examined for the initially unconstrained spherical membrane. We are particularly interested in examining the conditions for the onset of sustained oscillatory behavior.

\section{EQUATION OF MOTION}

In this section, the derivation of the governing equation of the motion of a spherical membrane under internal pressure is briefly reviewed for completeness. Although the equations for static inflation are well known [Beatty (1987)], their dynamic counterparts are not as extensively used. The classical Mooney strain energy function is used to described the rubber-like material.

Consider a spherical membrane of elastic, isotropic, and incompressible material of density $\rho_{0}$. The membrane deformation is described in spherical coordinates $(R, \Theta, \Phi)$ in the undeformed state, and $(r, \theta, \varphi)$ in the deformed state. Let $\left(R_{0}, H_{0}\right)$ and $\left(r_{0}, h_{0}\right)$ denote the membrane midsurface radius and thickness in the undeformed state and in the deformed state, respectively.

Let $\sigma_{r r}$ and $\sigma_{\theta \theta}=\sigma_{\phi \phi}$ be the diagonal components of the Cauchy stress tensors in the radial and circumferential directions, respectively. Because of the spherical symmetry, all variables depend only on the current radial coordinate $r$ and time $t$. In this case, the governing equation of interest is the conservation of momentum equation in the radial direction:

$$
\rho_{0} \ddot{r}=\frac{\partial \sigma_{r r}}{\partial r}+\frac{2}{r}\left(\sigma_{r r}-\sigma_{\theta \theta}\right) .
$$

Let $\lambda_{1}=h_{0} / H_{0}$ denote the principal stretch ratio in the thickness direction, and $\lambda_{2}=\lambda_{3}=\lambda=r_{0} / R_{0}$ be the principal stretch ratios in the circumferential directions. In this case, the incompressibility assumption implies that $\lambda_{1} \lambda^{2}=1$. Let $\Delta P$ denote the imposed pressure difference. Integration of Eq. (1) through the thickness, and use of the membrane approximation and the expressions for the stretch ratios lead to the following reduced form [Eringen and Suhubi (1974)]:

$$
\rho_{0} H_{0} R_{0} \ddot{\lambda}=\Delta P \lambda^{2}+\frac{2 H_{0}}{R_{0}} \frac{1}{\lambda}\left(\sigma_{r r}-\sigma_{\theta \theta}\right) .
$$

The expression for the stress difference $\sigma_{r r}-\sigma_{\theta \theta}$ will be determined through the constitutive relation between stress and strain (principal stretch ratio).

In the present work, the material studied is assumed to obey the Mooney-Rivlin constitutive equation [Mooney (1940); Treloar (1975)]. The corresponding expression for $\sigma_{r r}-\sigma_{\theta \theta}$ is given in terms of the principal circumferential stretch ratio $\lambda$ and the material constants $C$ and $\alpha$ as [Green and Adkins (1960)] 


$$
\sigma_{r r}-\sigma_{\theta \theta}=-2 C\left(\lambda^{2}-\frac{1}{\lambda^{4}}\right)\left(1+\alpha \lambda^{2}\right) .
$$

The differential equation (2) becomes

$$
\rho_{0} H_{0} R_{0} \ddot{\lambda}=\Delta P \lambda^{2}+\frac{4 C H_{0}}{R_{0}}\left(\frac{1}{\lambda^{5}}-\lambda\right)\left(1+\alpha \lambda^{2}\right) .
$$

To simplify the discussion on the influence of the different parameters in Eq. (4), we define the reduced time and pressure by

$$
\begin{gathered}
\tau=t \frac{2}{R_{0}} \sqrt{\frac{C}{\rho_{0}}}, \\
\Delta p=\Delta P \frac{R_{0}}{4 C H_{0}} .
\end{gathered}
$$

Equation (4) takes the following nondimensional form:

$$
\ddot{\lambda}=\Delta p \lambda^{2}+\left(\frac{1}{\lambda^{5}}-\lambda\right)\left(1+\alpha \lambda^{2}\right),
$$

in which $\lambda$ is a function of the reduced time variable $\tau$. Now a dot denotes differentiation with respect to $\tau$.

\section{DYNAMIC BEHAVIOR}

The dynamic inflation is now examined by solving Eq. (7) for a constant inflation pressure step. However, it is helpful to consider first the static inflation of a spherical membrane, which will be referred to later when dynamic inflation is analyzed.

\section{A. Static inflation}

In Eq. (7), the acceleration term is set to zero, and the inflation pressure step becomes an explicit function of the material parameter $\alpha$ and the principal circumferential stretch ratio $\lambda$ :

$$
\Delta p=\left(\frac{1}{\lambda}-\frac{1}{\lambda^{7}}\right)\left(1+\alpha \lambda^{2}\right) .
$$

The curves $\Delta p$ vs $\lambda$ are presented in Fig. 1 for three different values of $\alpha$ : $0 ., 0.1$, and 0.25 . The three curves are typically representative of three possible behaviors [Beatty (1987)].

The curve $\alpha=0$ represents the behavior of a neo-Hookean membrane. The maximum pressure the membrane can withstand in static inflation is called the critical static pressure or limit pressure, and is denoted $\Delta p_{S}^{C}$. For $0<\Delta p<\Delta p_{S}^{C}$, Eq. (8) has two distinct real roots, one on the ascending portion of the curve (for $\lambda<\lambda_{S}^{C}$, where $\lambda_{S}^{C}$ corresponds to $\left.\Delta p_{S}^{C}\right)$ and another on the descending portion $\left(\lambda>\lambda_{S}^{C}\right)$. For $\Delta p>\Delta p_{S}^{C}$, there is no real solution for Eq. (8): the membrane cannot withstand such an inflating pressure. For $\Delta p=\Delta p_{S}^{C}$, there is a double root, and for $\Delta p=0$, there are two real distinct roots at 0 and $+\infty$. The curve $\alpha=0.1$ is representative of the situation in which the inflation curve always presents two stable and one unstable parts. This is the case in which $0<\alpha<\alpha_{S}^{\lim }$, with $\alpha_{S}^{\lim } \simeq 0.214$. Here, the curve $\Delta p$ vs $\lambda$ consists of 


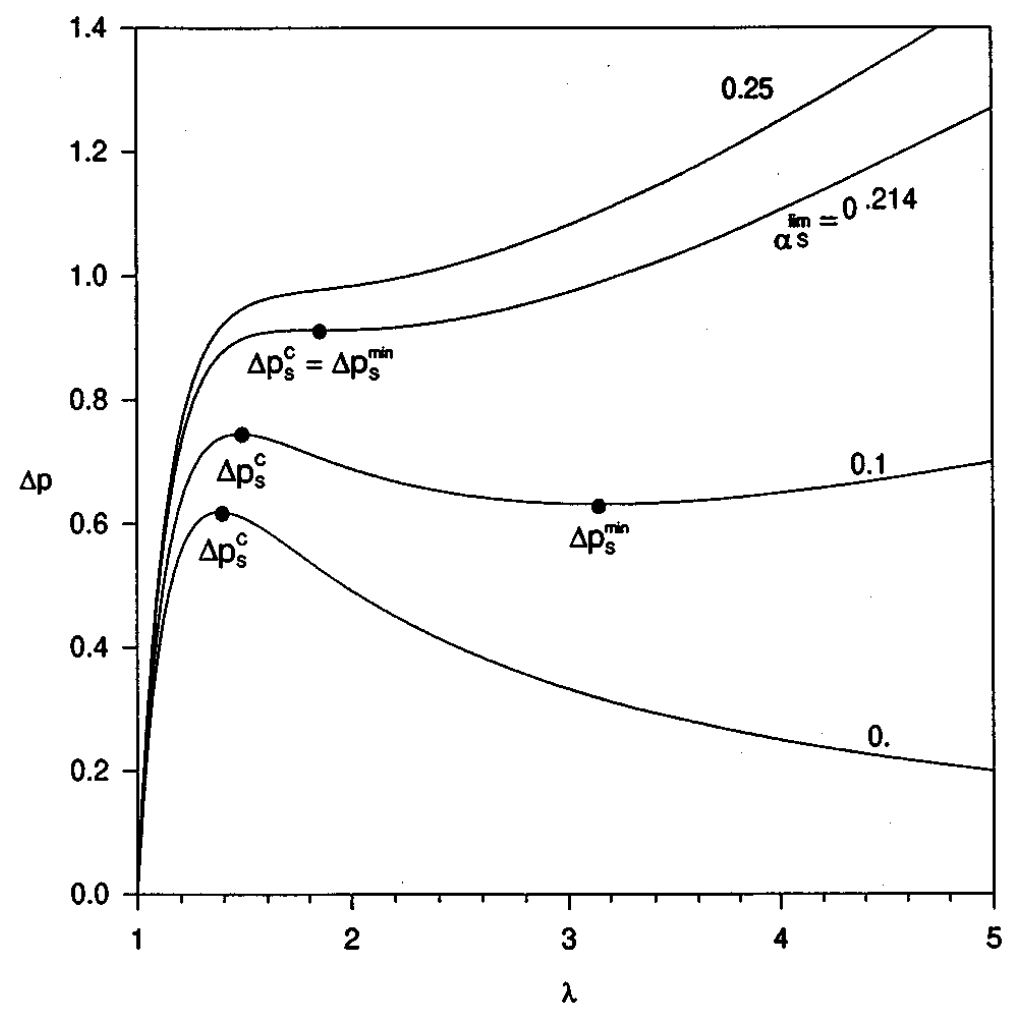

FIG. 1. Static inflation: normalized pressure step vs circumferential stretch ratio for different values of $\alpha$ shown on each curve.

three branches. The curve has two local extrema, one maximum for $\Delta p=\Delta p_{S}^{C}$ (this is not a critical value, because the membrane can withstand greater pressures) at $\lambda=\lambda_{S}^{C}$ and one minimum for $\Delta p=\Delta p_{S}^{\min }$ at $\lambda=\lambda_{S}^{\min }$. From Fig. 1, it can be seen that for $0<\Delta p<\Delta p_{S}^{\min }$, Eq. (8) has only one real root in the first stable branch. For $\Delta p_{S}^{\min }<\Delta p<\Delta p_{S}^{C}$, Eq. (8) has three real roots, one in the first stable branch, one in the unstable branch, and one in the second stable branch. For greater pressures $\left(\Delta p>\Delta p_{S}^{C}\right)$, Eq. (8) presents only one real root in the second stable branch. It is to be noted that for $\Delta p=\Delta p_{S}^{\min }$ and $\Delta p=\Delta p_{S}^{C}$ there are only two real roots. In Fig. 1, the curve $\alpha=0.25$ stands for all the cases where $\alpha>\alpha_{S}^{\lim }$. All the curves are monotonically increasing and the function (8) is a bijection: for every inflating pressure step $\Delta p$, there is only one real root.

\section{B. Dynamic inflation}

Equation (7) is rewritten as a set of two differential equations of the first order:

$$
\begin{gathered}
\dot{\lambda}=\mathrm{v} \\
\dot{\mathrm{v}}=\Delta p \lambda^{2}+\left(\frac{1}{\lambda^{5}}-\lambda\right)\left(1+\alpha \lambda^{2}\right) .
\end{gathered}
$$

The initial conditions are:

$$
\begin{aligned}
& \lambda(\tau=0)=\lambda_{\text {init }}, \\
& \mathrm{v}(\tau=0)=\mathrm{v}_{\text {init }},
\end{aligned}
$$




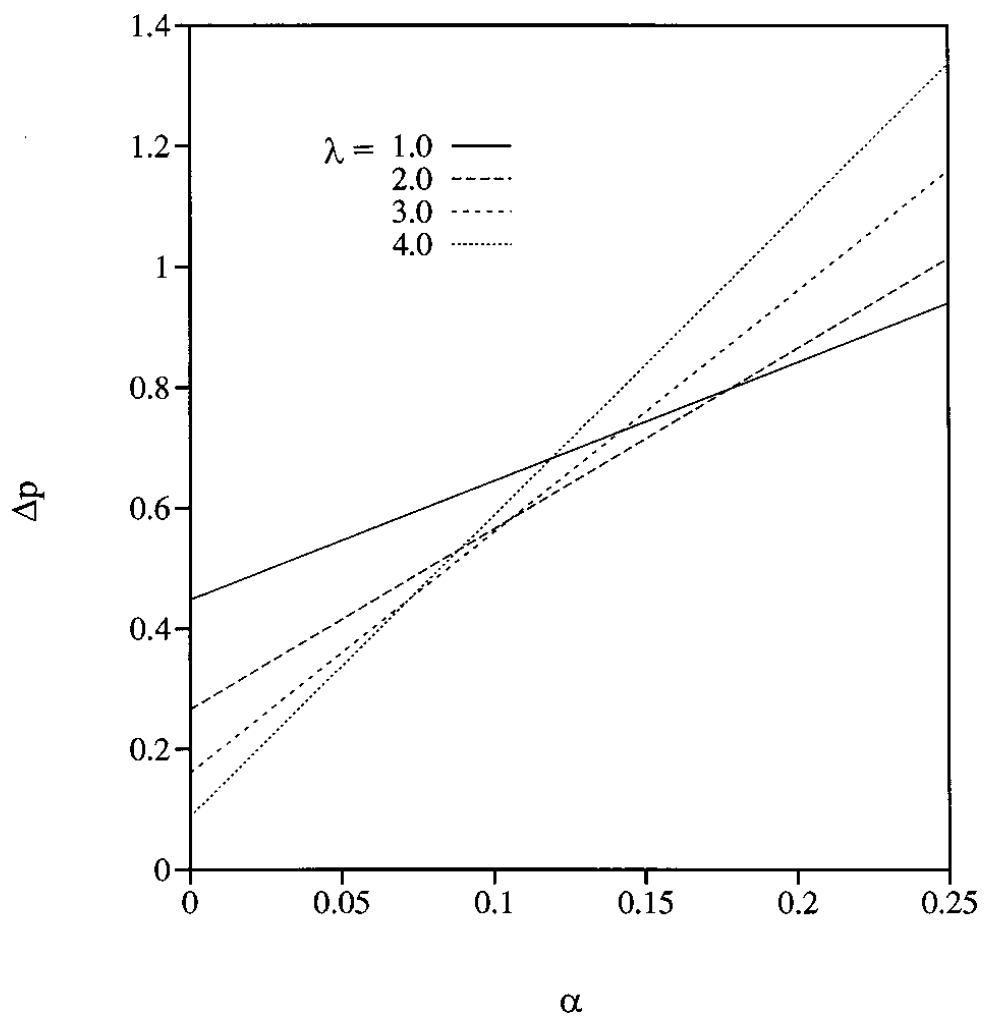

FIG. 2. Codimension-two bifurcation: normalized pressure step vs $\alpha$ for different values of the circumferential stretch ratio $\lambda$.

where $\lambda_{\text {init }}$ and $v_{\text {init }}$ will be given when specific examples are covered. With these initial conditions, problem (9) and (10) is well posed and has a unique solution. Of course, given the nonlinearities present, the problem can admit a multiple-valued solution. However, the problem is deterministic, and only one solution corresponds to a set of initial conditions. The equations are solved by using a sixth-order Runge-Kutta method (subroutine IVPRK from the IMSL library).

The dynamical system (9) is codimension 2 since two parameters are required for a bifurcation to occur. In this case, the two parameters are $\Delta p$ and $\alpha$. The coexistence of fixed points is illustrated in Fig. (2), which shows constant $\lambda$ curves in the $(\Delta p, \alpha)$ plane. It is clear from Fig. 2 that a given stretch ratio may be maintained by an infinite number of combinations of the two parameters (each $\lambda$ curve intersects an infinite number of other curves). More importantly, Fig. 2 shows that two different values of the stretch ratio can correspond to the same value of $\Delta p$ and $\alpha$.

Since $\Delta p$ is independent of time, $\mathrm{v}$ can be obtained as an explicit function of $\lambda$ by taking the ratio of the two equations system (9) and integrating from $\left(\lambda_{\text {init }}, v_{\text {init }}\right)$ to $(\lambda, v)$. We thus obtain the equation of energy conservation for the inflation of the spherical membrane [Dym (1974)]:

$$
\begin{aligned}
\frac{1}{2} \mathrm{~V}^{2}-\frac{1}{2} \mathrm{~V}_{\text {init }}^{2}= & \Delta p\left(\frac{\lambda^{3}}{3}-\frac{\lambda_{\text {init }}^{3}}{3}\right)-\left[\frac{\lambda^{2}}{2}+\frac{1}{4 \lambda^{4}}+\alpha\left(\frac{\lambda^{4}}{4}+\frac{1}{2 \lambda^{2}}\right)\right] \\
& +\left[\frac{\lambda_{\text {init }}^{2}}{2}+\frac{1}{4 \lambda_{\text {init }}^{4}}+\alpha\left(\frac{\lambda_{\text {init }}^{4}}{4}+\frac{1}{2 \lambda_{\text {init }}^{2}}\right)\right] .
\end{aligned}
$$


In Eq. (11), the left-hand side is the change in kinetic energy, the first term on the right-hand side is the work done by the inflating pressure, and the last terms represent the elastic strain energy stored by the membrane. This equation allows us to directly obtain the phase curves $v$ vs $\lambda$. We next examine the linear stability of the static (steady-state) solution.

\section{Linear stability analysis}

Assuming that $\alpha$ and $\Delta p$ are fixed, the equilibrium states are the points that satisfy $\dot{\lambda}=0$ and $\dot{v}=0$ in system (9). These points are the couples $\left(\lambda_{e}, \mathrm{v}_{e}\right)$ of the phase curves where $\lambda_{e}$ satisfies Eq. (8) and $\mathrm{v}_{e}=0$. The number of such points depends on the values of $\alpha$ and $\Delta p$ and was investigated in detail in Sec. III A.

In order to determine the stability of each equilibrium state, we must study the behavior of system (9) about this state. The Jacobian matrix $[J]$ of system (9) at the equilibrium point $\left(\lambda_{e}, 0\right)$ is given by

$$
[J]_{\lambda=\lambda_{e}, \mathrm{v}=0}=\left[\begin{array}{ll}
0 & 1 \\
\Omega & 0
\end{array}\right],
$$

in which

$$
\Omega=2 \Delta p \lambda_{e}-\left(\frac{5}{\lambda_{e}^{6}}+1\right)-\alpha\left(\frac{3}{\lambda_{e}^{4}}+3 \lambda_{e}^{2}\right) .
$$

Let $\omega_{1}$ and $\omega_{2}$ be the two eigenvalues of the problem, which dictate the approximate form of $\lambda$ and $v$ in the neighborhood of $\left(\lambda_{e}, 0\right)$. The two roots $\omega_{1}$ and $\omega_{2}$ depend on the sign of $\Omega$ :

(i) If $\Omega>0$, the two roots are real and of opposite signs, $\omega_{1}=-\sqrt{\Omega}$ and $\omega_{2}=\sqrt{\Omega}$.

(ii) If $\Omega<0$, the two roots are purely imaginary, $\omega_{1}=-i \sqrt{-\Omega}$ and $\omega_{2}=i \sqrt{-\Omega}$.

(iii) If $\Omega=0$, zero is the double root, $\omega_{1}=\omega_{2}=0$.

Let us now examine the dependence of $\Omega$ (more importantly, the sign of $\Omega$ ) on $\Delta p$ and $\alpha$ [recall that $\lambda_{e}$ is determined from Eq. (8)]. If $\Omega>0$, the equilibrium point $\left(\lambda=\lambda_{e}, \mathrm{v}=0\right)$ is unstable and is a saddle point; if $\Omega<0, \lambda(t)$ and $\mathrm{v}(t)$ are periodic in the neighborhood of $\left(\lambda=\lambda_{e}, v=0\right)$, which is an elliptic center. The number and stability of the equilibrium branches depend on $\Delta p$ and $\alpha$. If we note that

$$
\left.\frac{d \Delta p}{d \lambda}\right|_{\lambda=\lambda_{e}}=-\frac{\Omega}{\lambda_{e}^{2}},
$$

then we can clearly see that if $\Omega<0$, the ascending branch(es) of a given static curve in Fig. 1 is (are) stable. If $\Omega>0$, the descending branch of the static curve is unstable, which confirms previous stability findings [Alexander (1971)]. [Figure 1 also shows the span of stable and unstable branches with the limit points in $\Delta p$ corresponding to the extrema in the static curves.

Linear stability analysis is limited to small departures from equilibrium. Before analyzing the nonlinear dynamical behavior, it is helpful to examine the solution in the $(\lambda, v)$ phase plane for the three values of the parameter $\alpha$ as in Fig. 1. The resulting phase diagrams are shown in Fig. 3. For $\alpha=0$, if $0<\Delta p<\Delta p_{S}^{C}$, there are two equilibrium points: one elliptic center $\left(\lambda_{\mathrm{V}}, 0\right)$ and one saddle point $\left(\lambda_{S}, 0\right)$ with $\lambda_{\mathrm{V}}<\lambda_{S}$. The corre- 

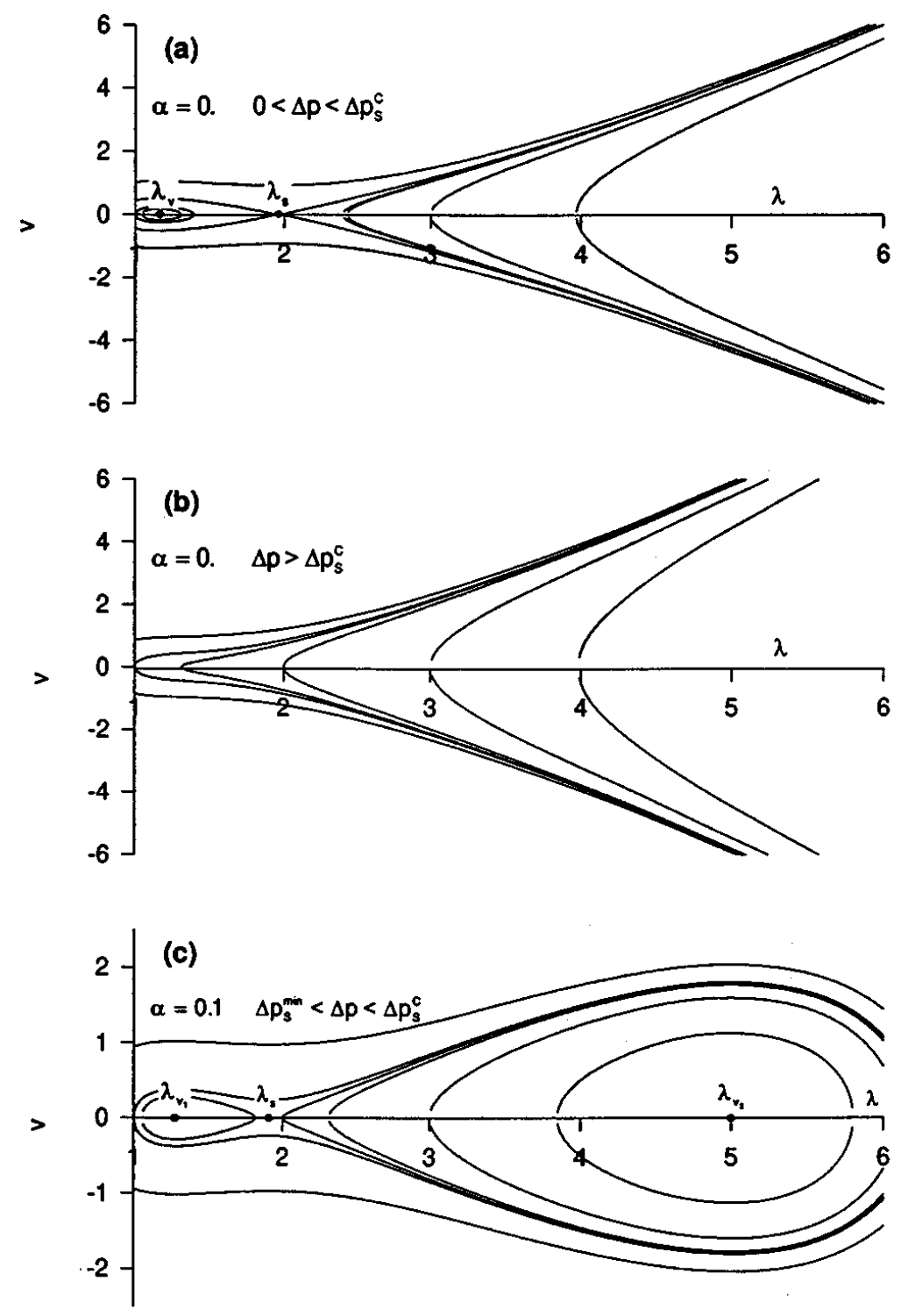

FIG. 3. Phase-plane trajectories $\mathrm{v}$ vs $\lambda$ for three typical ranges of $\alpha$ and $\Delta p$ and general initial conditions $\left(\lambda_{\text {init }}, \mathrm{v}_{\text {init }}\right)$.

sponding $(\lambda, \mathrm{v})$ trajectories are shown in Fig. 3(a). There are closed curves in the vicinity of the elliptic center, and these curves become open branches in the neighborhood of the saddle point. Depending on the initial conditions $\left(\lambda_{\text {init }}, \mathrm{v}_{\text {init }}\right)$, the solution may oscillate around the elliptic center or grow exponentially away from the saddle point. Figure 3(a) displays a global homoclinic (separatrix) solution confining the area of periodic solutions about the stable center and diverging solutions outside; note the similarity with Hamiltonian problems with "escape from a potential well" [Guckenheimer and Holmes (1983)]. The homoclinic solution is obtained from Eq. (11) using the initial conditions of the saddle. Figure 3(b) displays the trajectories corresponding to $\Delta p>\Delta p_{S}^{C}$. Regardless of the initial conditions, the solution does not converge to a fixed point. This is, of course, resulting from the fact that there are no real solutions to Eq. (8). The phase plane trajectories in the presence of three fixed points are presented in Fig. 3(c) for $\alpha=0.1$ and $\Delta p_{S}^{\min }<\Delta p<\Delta p_{S}^{C}$. In this case, there are two elliptic centers $\left(\lambda_{\mathrm{v}_{1}}, 0\right)$ and $\left(\lambda_{\mathrm{v}_{2}}, 0\right)$ and one saddle point $\left(\lambda_{s}, 0\right)$ with $\lambda_{\mathrm{v}_{1}}<\lambda_{s}<\lambda_{\mathrm{v}_{2}}$. Depending on the values of the initial conditions, the trajectories can be two closed curves, each one of them around one elliptic center or only one closed curve that surrounds all three fixed points. Figure 3(c) shows 
also the case of a periodic limit orbit that surrounds the three fixed points. In the former case, the solution tends to oscillate around one elliptic center or the other. In the latter case, the solution remains periodic but oscillates around both elliptic centers over one period of time. The trajectory turns around one of the elliptic centers, then enters the saddle-point neighborhood and goes on around the other elliptic center. For $\Delta p$ $\in\left[0, \Delta p_{S}^{\min }[U] \Delta p_{S}^{C},+\infty[\right.$, there is only one fixed point, which is an elliptic center. Thus, the trajectories (not shown) are closed curves centered on this point, regardless of the initial conditions. Figure 3(c) thus exhibits two homoclinic solutions confining (internal) periodic solutions about the centers and a set of (external) periodic solutions about both homoclinic solutions. This situation is similar to asymmetric double-well problems. Note that for $\alpha=0.25$ and for any value of $\Delta p$, there is only one fixed elliptic center.

\section{Nonlinear dynamic inflation}

In the previous section, we examined the phase-plane trajectories for general initial conditions. We determined the number of fixed points and analyzed their stability as function of the control parameters $\alpha$ and $\Delta p$. Consider now the special case of a membrane initially in equilibrium subject to the following initial conditions $\left(\lambda_{\text {init }}=1\right.$, $v_{\text {init }}=0$ ). This corresponds to a spherical membrane of a given radius initially in equilibrium.

We first examine the conditions for a periodic response. Obviously, when the motion of the membrane is periodic, the corresponding phase curve is a closed loop in the $(\lambda, v)$ plane. Noting that the initial point $(1,0)$ is an intersection point between the $v=0$ axis and the phase trajectory, it can be inferred that this trajectory must intersect the $v=0$ axis at least one more time for it to be a closed loop. If $v$ is set equal to zero in Eq. (11), the relation between $\Delta p$ and $\lambda$ becomes

$$
\Delta p=\left\{\begin{array}{l}
\left(\frac{3}{\lambda^{3}-1}\right)\left[\frac{\lambda^{2}}{2}+\frac{1}{4 \lambda^{4}}+\alpha\left(\frac{\lambda^{4}}{4}+\frac{1}{2 \lambda^{2}}\right)-\frac{3}{4}(1+\alpha)\right] \quad(\lambda \neq 1) \\
0 \quad(\lambda=1)
\end{array} .\right.
$$

The curves $(\lambda, \Delta p)$, corresponding to Eq. (15), are presented in Fig. 4, together with the static curves based on Eq. (8), for different values of $\alpha$. Thus, the solid curves in Fig. 4 correspond to the loci of solutions with zero velocity $(\mathrm{v}=0)$ and nonzero acceleration $(\dot{v} \neq 0)$, whereas the dashed (static) curves correspond to the loci of solutions with both $v=0$ and $\dot{v}=0$. It is clear from Fig. 4 that the overall behavior of the $(\lambda, \Delta p)$ curves is the same for both static and dynamic inflations. There is a value of the material parameter, $\alpha_{D}^{\lim } \simeq 0.145$, beyond which, similarly to $\alpha_{S}^{\lim }, \Delta p$ increases monotonically with $\lambda$. The $\alpha=0$ curve has one maximum corresponding to a critical pressure value, $\Delta p_{D}^{C}$. For $0<\alpha<\alpha_{D}^{\lim }$, there are three branches, a local maximum $\Delta p_{D}^{C}$, and a local minimum $\Delta p_{D}^{\min }$, as in the static case. Note that the values of the critical parameters are highly dependent on the initial conditions. It is interesting to note that $\alpha_{D}^{\lim }<\alpha_{S}^{\lim }$ and $\Delta p_{S}^{\min }<\Delta p_{D}^{\min }<\Delta p_{D}^{C}<\Delta p_{S}^{C}$ independently of the value of $\alpha$.

It is useful to closely examine the interplay between the static and dynamic behaviors through the corresponding curves, since the static curves represent equilibrium regions towards which the dynamic solution may eventually be attracted in real systems (with damping). There are four important ranges of values for $\alpha$. Recall that in static inflation three main ranges of interest were examined: $\alpha=0,0<\alpha<\alpha_{S}^{\lim }$, and $\alpha>\alpha_{S}^{\lim }$. In the dynamic case, we identify four regions of interest, namely, $\alpha=0,0<\alpha<\alpha_{D}^{\lim }$, 


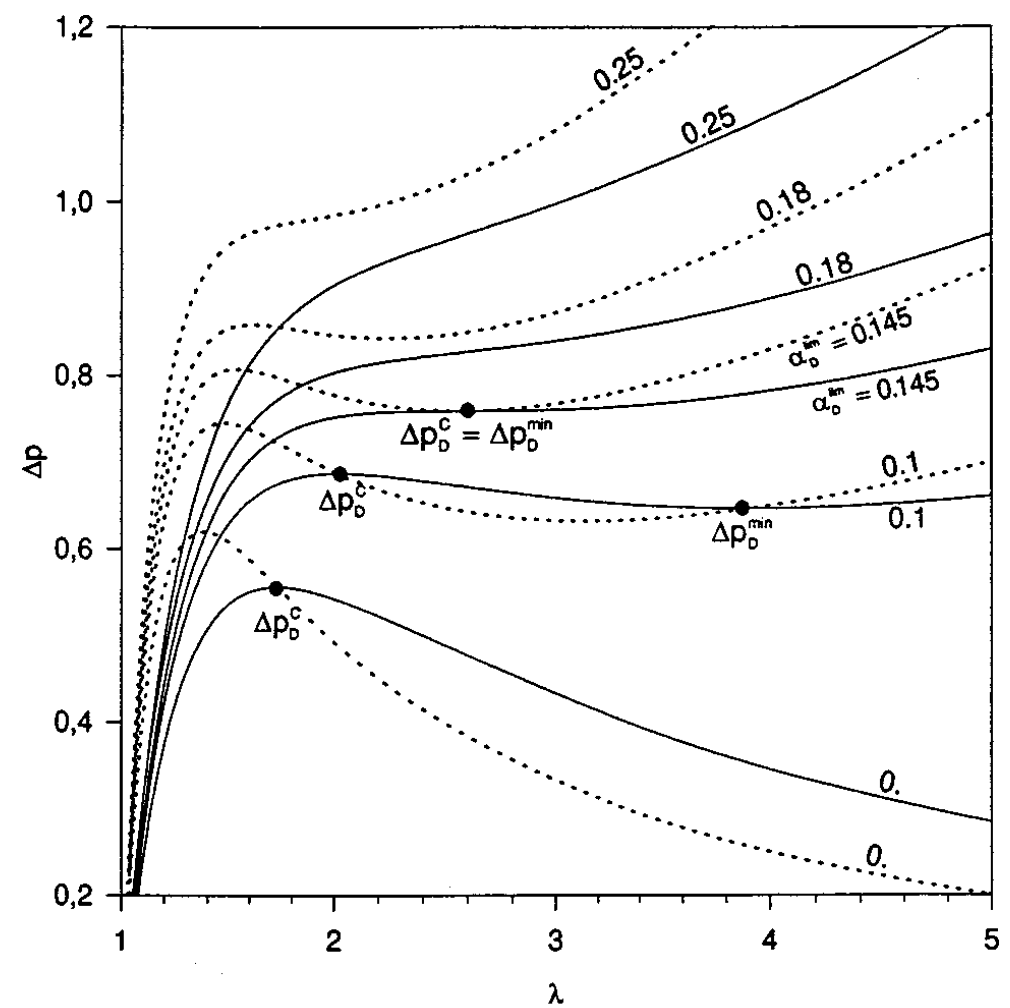

FIG. 4. $\Delta p$ vs $\lambda$ for static ( $\cdots)$ and dynamic (--) inflations (the corresponding value of $\alpha$ is shown on each curve).

$\alpha_{D}^{\lim }<\alpha<\alpha_{S}^{\lim }$, and $\alpha>\alpha_{S}^{\lim }$. We are particularly interested in the conditions under which oscillatory movement occurs; we must then determine the location of the first intersection point $\lambda_{i}\left(\lambda_{i}>1\right)$, between a dynamic curve and a horizontal line, representing the value of a given pressure step $\Delta p$. Note that the same horizontal line intersects the static curve (corresponding to the same $\alpha$ value) at $\lambda_{e}$ (there may be more than one intersection point). The solution may thus end up oscillating between $\lambda=1$ and $\lambda_{i}$ around $\lambda_{e}$.

We now focus our discussion on the range $\alpha>0.1$. Figures 5 and 6 display the resulting dynamics for two different values of $\alpha . \alpha=0.18$ and 0.25 , respectively, with initial conditions $\lambda_{\text {init }}=1, v_{\text {init }}=0$. Each figure shows (a) the dynamic and static pressure curves together with the intersecting points $\lambda_{i}$ 's and $\lambda_{e}$ 's for a typical level of inflating pressure, (b) the phase-plane trajectories for various levels of inflating pressure, and (c) the corresponding temporal behaviors for $\lambda$. Typically, there are four different $\Delta p$ ranges that can be identified. For $0<\Delta p<\Delta p_{D}^{C}$, there are generally two intersection points, $\lambda_{i_{1}}$ and $\lambda_{i_{2}}\left(\lambda_{i_{1}}<\lambda_{i_{2}}\right)$, between the dynamic curve and the horizontal line, and two intersecting (equilibrium) points, $\lambda_{e_{1}}$ and $\lambda_{e_{2}}\left(\lambda_{e_{1}}<\lambda_{e_{2}}\right)$, for the static curve. In this case, the membrane oscillates between the unconstrained (initial) state and $\lambda_{i_{1}}$ around the stable static equilibrium (elliptic) point $\lambda_{e_{1}}$. For the particular case $\Delta p=\Delta p_{D}^{C}$, there is only one intersection point $\lambda_{i}$ and still two intersection points $\lambda_{e_{1}}$ and $\lambda_{e_{2}}$. This is, in fact, a degenerate case where the two roots of Eq. (15) are equal to $\lambda_{i}$. Note also that $\lambda_{i}$ is equal to $\lambda_{e_{2}}$, this latter being a saddle point. In this case, the membrane neither oscillates nor inflates any further but maintains a static (unstable) equilibrium state. This corresponds to an oscillation with an infinite period. For 

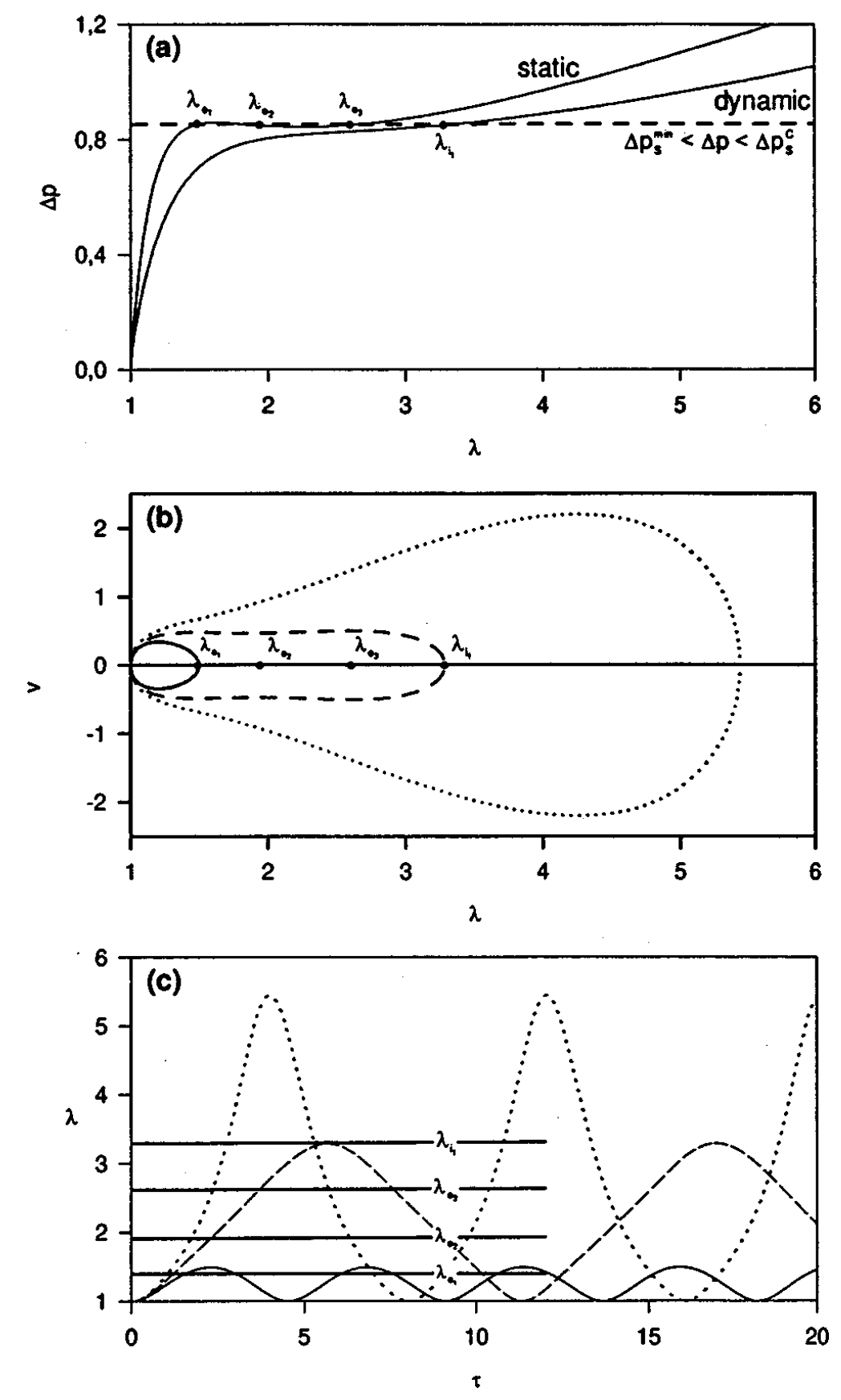

FIG. 5. $\alpha=0.18$ : (a) $\Delta p$ vs $\lambda$ for static and dynamic inflations, (d) stable phase-plane trajectories corresponding to $\lambda_{\text {init }}=1$ and $\mathrm{v}_{\text {init }}=0$, and (c) temporal behavior of the circumferential stretch ratio $\lambda$, for four $\Delta p$ ranges: (-) $0<\Delta p<\Delta p_{D}^{C},(--) \Delta p=\Delta p_{D}^{C},(\cdots) \Delta p_{D}^{C}<\Delta p<\Delta p_{S}^{C}$, and (-..) $\Delta p \geqslant \Delta p_{S}^{C}$.

$\Delta p_{D}^{C}<\Delta p<\Delta p_{S}^{C}$, the phase trajectory is reduced to an open curve. The membrane tends to inflate indefinitely, but because of the presence of the saddle point the phaseplane trajectory exhibits a local minimum in the neighborhood of the saddle point. For $\Delta p \geqslant \Delta p_{S}^{C}$, there are no intersection points at all and the phase-plane trajectory increases monotonically with $\lambda$.

Consider now the case when $\alpha_{D}^{\lim }<\alpha<\alpha_{S}^{\lim }$, more precisely, when $\alpha=0.18$. Figure 5 shows the behavior for only three ranges of inflating pressure, since the dynamic curve exhibits a strictly monotonic behavior [Fig. 5(a)]. Note that in this case the static curve still exhibits two extrema. The phase-plane trajectory is always a closed cycle but its shape depends on the value of $\Delta p$ relative to the extrema of the static curve. For 

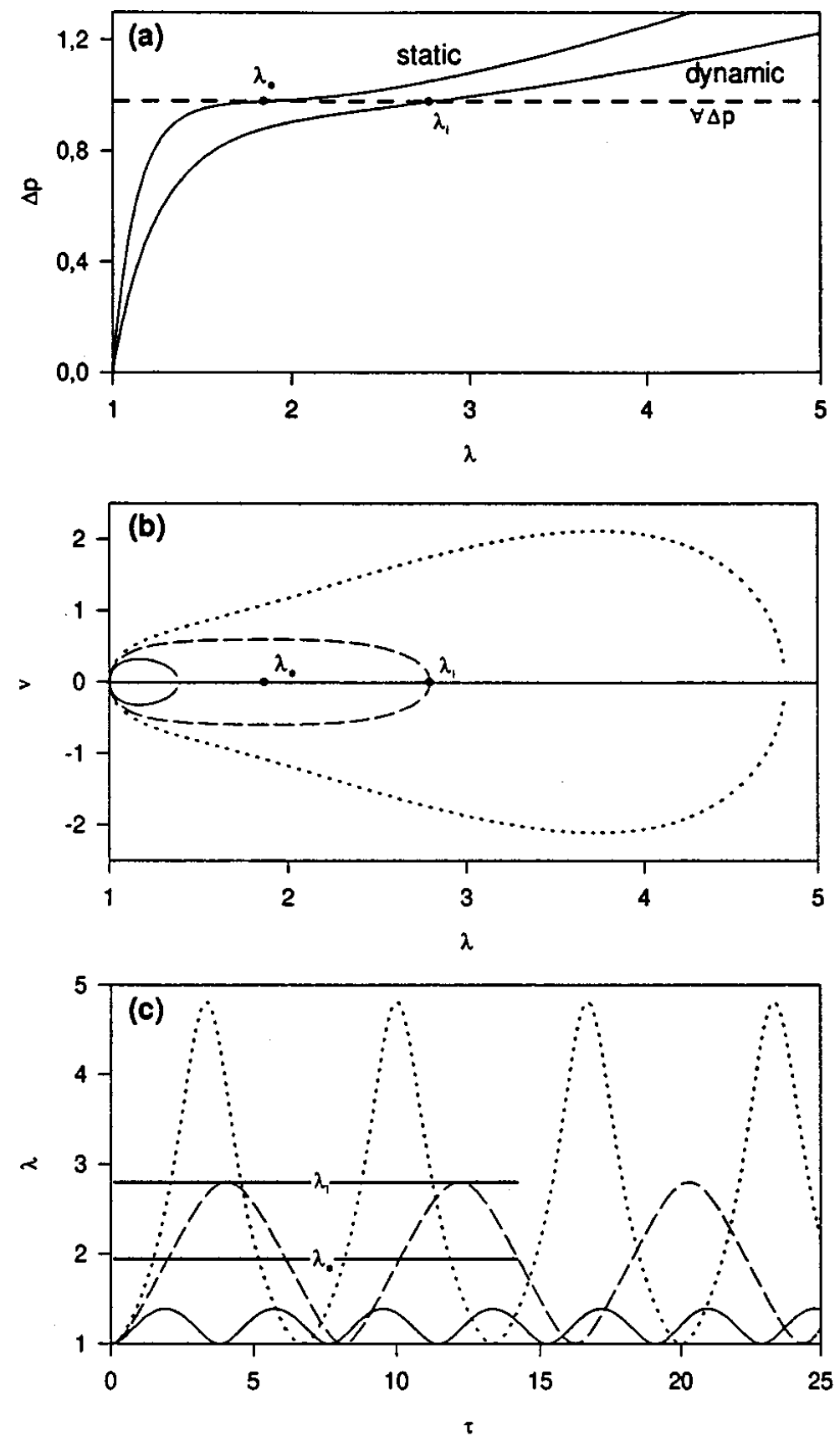

FIG. 6. $\alpha=0.25$ : (a) $\Delta p$ vs $\lambda$ for static and dynamic inflations, (b) stable phase-plane trajectories corresponding to $\lambda_{\text {init }}=1$ and $\mathrm{v}_{\text {init }}=0$, and (c) temporal behavior of the circumferential stretch ratio $\lambda$, for three $\Delta p$ ranges: $(-) 0<\Delta p<\Delta p_{S}^{\min },(--) \Delta p_{S}^{\min }<\Delta p<\Delta p_{S}^{C}$, and $(\cdots) \Delta p \geqslant \Delta p_{S}^{C}$.

$\Delta p<\Delta p_{S}^{\min }$, there is only one equilibrium point on the first stable branch of the static curve and the membrane oscillates around it. For $\Delta p_{S}^{\min }<\Delta p<\Delta p_{S}^{C}$, there are three equilibrium points, and the membrane oscillates around the two vortices as shown in Figs. 5(b) and 5(c). Note that the necking is insignificant in this case since the motion amplitude is relatively small.

For $\alpha=0.25$, both the dynamic and static curves are strictly monotonic. In this case, condition (15) has only one root and there is always one equilibrium point. The membrane always oscillates. Therefore, the period of motion remains finite. Figure 6 shows the corresponding behavior for three different values of the pressure step. 


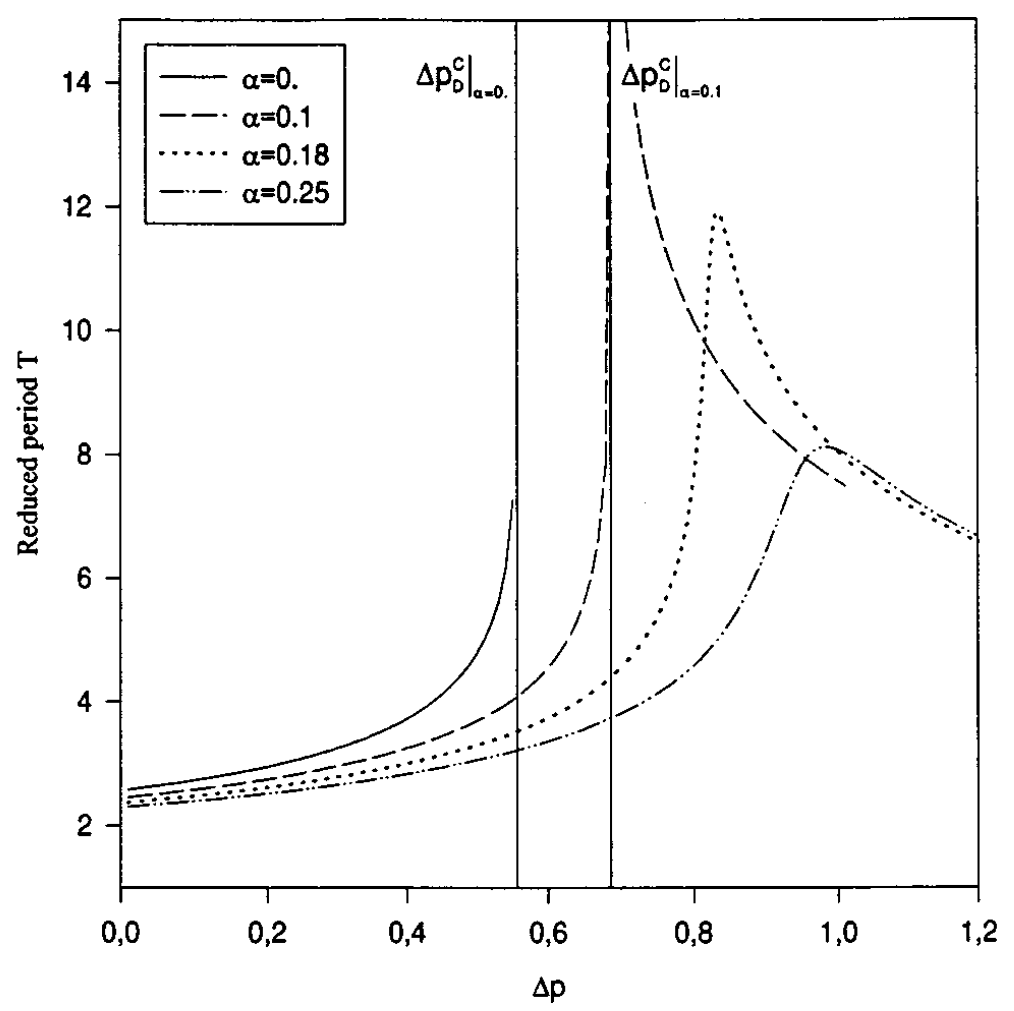

FIG. 7. Influence of the pressure step on the period of motion for different values of $\alpha$.

After having determined the ranges of values for $\alpha$ and $\Delta p$ for which oscillatory inflation occurs, we next examine the influence of these two parameters on the period of oscillation. It was found above that the membrane tends to oscillate in most cases of practical interest, and that the amplitude of the motion depends on the value of the pressure step (for given $\alpha$ ). Similarly, Figs. 5 and 6(c) show that the period of oscillation is closely related to $\Delta p$. Figure 7 shows the dimensionless period $T$ as function of $\Delta p$ for the four values of $\alpha$ considered above. Generally, the period of oscillation tends to increase at small inflating pressure, reaches a maximum value and then decreases in the higher $\Delta p$ range. The only case in which the membrane becomes unstable is when $\alpha=0$ (neo-Hookean material) and $\Delta p>\Delta p_{D}^{C}$. For $\alpha=0$ and $\Delta p=\Delta p_{D}^{C}$, and $\alpha=0.1$ (representing the case $0<\alpha<\alpha_{S}^{\lim }$ ) and $\Delta p=\Delta p_{D}^{C}$, the membrane tends to reach an unstable equilibrium (saddle) point and the period goes to infinity. After this point, for $\alpha=0.1$ and $\Delta p>\Delta p_{D}^{C}$, the membrane oscillates again and the period of motion decreases monotonically. In all the other situations, the membrane oscillates. But, because of the nonlinearity of the differential system, it is not enough to conclude that the period of motion grows as the pressure step grows [see Figs. 5 and 6(c)]: for $\alpha=0.25$ (representing $\alpha>\alpha_{S}^{\lim }$ ), the motion is never unstable but the period does not increase monotonically with pressure. The corresponding curve $T$ vs $\Delta p$ admits a maximum. For $\alpha=0.18$, the results are similar to the latter case, because $\alpha$ is also greater than $\alpha_{S}^{\lim }$. This last result differs from Akkas (1978) who concluded that, for $\alpha>\alpha_{S}^{\lim }$, as $\Delta p$ gets larger, the period of oscillations also gets larger. This difference may be due to his hypothesis of constant thickness of the membrane during inflation.

\section{DISCUSSION AND CONCLUSION}

There are two major issues regarding the class of materials targeted in this study that we would like to discuss in some detail. The first is the use of the Mooney-Rivlin model 
as opposed to using other elastic models. The second is the fact that the material in question does not include any viscous damping. Thus, it is important to point out the limitations and advantages of the assumptions adopted here. Hyperelastic models, including the Mooney-Rivlin model, can capture some of the phenomena observed during the (primary) inflation stage in a wide range of forming processes. Beatty (1987) compared the normalized pressure-stretch curve obtained from the experimental inflation of a spherical balloon against results based on the Mooney-Rivlin model. He found that, while the normalized pressure grows indefinitely with stretch, the slope of the MooneyRivlin curve approaches a constant value for large stretch. Hence, the Mooney-Rivlin model does not predict an indefinitely large, bursting pressure at a finite value of the stretch. This shortcoming of the Mooney-Rivlin model is by now well accepted, as the model can only be accurate for moderately large and not very large deformation. Nonetheless, according to Beatty (1987), "'the Mooney-Rivlin model does provide a satisfactory qualitative (and probably conservative) description of the overall primary balloon inflation phenomenon." Although the discrepancy between theory and experiment could be in part due to the fact that the balloon was not perfectly spherical during the experiment, and also to the assumption of incompressibility of the material applied throughout the analysis. Obviously, some additional and systematical work is needed to examine the effects of materials properties on inflation dynamics.

On the other hand, the present study should not be regarded as limited to the stability analysis of only Mooney-Rivlin materials, but as a study relevant to a large class of hyperelastic materials in the limit of moderately large strain. The methodology developed in the present study can be applied to other constitutive models, and the type and range of phenomenological behaviors examined are likely to be encountered in other hyperelastic materials. The Mooney-Rivlin model is related to or is the limit of many more realistic models for hyperelastic materials. These include the following models: generalized Rivlin [Rivlin (1948)], three-term model of Isihara et al. (1951), Rivlin-Saunders (1951), Gent-Thomas (1958), Biderman (1958), Carmichael-Holdaway (1961), HartSmith (1966), Valanis-Landel (1967), Alexander (1968), Ogden (1972), and BlatzSharda-Tschoegl (1974). These models are derived on the basis of phenomenological, statistical, or empirical arguments. Our own studies [Khayat and co-workers (1998); (1995); (1994a); (1994b); (1993); (1992)] on the modeling of blow-molding and thermoforming processes showed a remarkable usefulness for the Mooney-Rivlin model, for materials undergoing industrially complex transformations. See, in particular, Khayat and Derdouri (1995) and Derdouri et al. (1994) for comparison between theory and experiment. Similarly, other works also support this claim [see, for instance, Charrier et al. (1989) and Delorenzi and Nied (1990)].

Let us now turn to the second issue regarding the absence of damping in the model. In this regard, one may conclude hastily that the purely elastic model in this case is of no relevance to physical reality. It is true that damping will make the oscillatory solution decay to a fixed point. However, there are instances where transient and not only longterm behavior is of the main physical or practical interest. It is possible that the inflation process in question may be so short that sustained oscillations, which stem from dominant elastic effects, are observed during the whole process. In blow molding and thermoforming, for instance, the inflation stage may last only a few milliseconds, and viscous effects are negligible. Moreover, since the sustenance of oscillations depends on the effects of elasticity relative to viscous effects, it is the type of (polymer) material used that dictates the time it takes for oscillations to decay (relaxation time). In a previous study [Khayat and Garcia-Rejon (1992)] we used a highly nonlinear viscoelastic constitutive model to study the inflation of a spherical shell of a viscoelastic fluid. It was 
found that beyond a critical Deborah number, oscillatory (as opposed to monotonic) growth sets in. It was also found that the amplitude and frequency of oscillations depend strongly on the pressure history inside the shell; a sudden initial jump in pressure tends to enhance oscillatory behavior.

In conclusion, the dynamic inflation of a spherical membrane made of Mooney-Rivlin material and subjected to a uniform step pressure was extensively studied. The nature of singular points was determined by a linear stability analysis for different ranges of values of the material parameter and the pressure step. The problem of the dynamic inflation of an initially unconstrained membrane exhibits critical values of the material parameter $\alpha_{D}^{\lim }$ and of the pressure step, $\Delta p_{D}^{C}$ (counterparts of the classical static critical values $\alpha_{S}^{\lim }$ and $\Delta p_{S}^{C}$ ) [Alexander (1971); Beatty (1987)]. We examined, in particular, the conditions for the onset of oscillatory inflation around the (static) equilibrium points. It was found that oscillatory motion tends to set in for Mooney-Rivlin materials with a period that is maximum at some inflating pressure. It was also found that the period tends to decrease as the Mooney parameter ratio increases.

Although the equations governing the inflation of the spherical membrane are Hamiltonian (as they do not account for any damping), it is difficult to assess their physical or practical relevance. A more realistic model should include the effects of viscosity, leading to a viscoelastic solid or fluid model. The inflation of a viscoelastic liquid shell obeying the modified ZFD model was earlier examined by Khayat and Garcia-Rejon (1992). It is found that, for a pressure suddenly imposed initially, the shell grows exponentially with time as long as the Deborah number De is small. When De exceeds a critical level, the growth becomes oscillatory. However, the amplitude and frequency of oscillations is strongly influenced by the rate of internal pressure. Calculations show that the oscillations are essentially absent for a slowly increasing pressure (compared to an initial sudden step). Since the pressure, in practice, cannot be suddenly imposed, it is likely that oscillations do not emerge in reality as a result of the strong damping.

\section{References}

Akkas, N., "On the dynamic snap-out instability of inflated non-linear spherical membranes,' Int. J. NonLinear Mechan. 13, 177-183 (1978).

Alexander, H., "A constitutive relations for rubber-like materials," Int. J. Eng. Sci. 6, 549-563 (1968).

Alexander, H., "Tensile instability of initially spherical balloons,' Int. J. Eng. Sci. 9, 151-162 (1971).

Beatty, M. F., "Topics in finite elasticity: Hyperelasticity of rubber, elastomers, and biological tissues-with examples,' Appl. Mech. Rev. 40, 1699-1734 (1987).

Blatz, P., S. C. Sharda, and N. W. Tschoegl, "Strain energy function for rubberlike materials based on a generalized measure of strain," Trans. Soc. Rheol. 18, 145-161 (1974).

Carmichael, A. J. and H. W. Holdaway, "Phenomenological elastomechanical behavior of rubber over wide ranges of strain,’’ J. Appl. Phys. 32, 159-166 (1961).

Charrier, J. M., S. Shrivastava, and R. Wu, "Free and confined inflation of elastic membranes in relation to thermoforming-non-axisymmetric problems,'” J. Strain Anal. 24, 55-73 (1989).

DeLorenzi, H. G. and H. F. Nied, "Finite Element Simulation of Thermoforming and Blow Molding," in Modeling of Polymer Processing: Recent Developments, edited by A. I. Issayev (Hanser, 1990), pp. 117171.

Derdouri, A., R. E. Khayat, and A. Garcia-Rejon, "Study of the Simultaneous Extension and Inflation of PET Performs," in Computer-Aided Engineering of Polymer Processing: From Dream to Reality, edited by K. Hiemasekhar and V. Prasad (American Society of Mechanical Engineers (ASME), New York, 1994), pp. $321-326$.

Dym, C. L., Stability Theory and Its Applications to Structural Mechanics (Noordhoff International, Leyden, 1974).

Eringen, A. C. and E. S. Suhubi, Elastodynamics (Academic, New York, 1974). 
Gent, A. N. and A. G. Thomas, "Forms of the stored (strain) energy function in vulcanized rubber,' J. Polym. Sci. 28, 625-637 (1958).

Green, A. E. and J. E. Adkins, Large Elastic Deformations and Nonlinear Continuum Mechanics (Clarendon, Oxford, 1960).

Guckenheimer, J. and P. Holmes, Nonlinear Oscillations, Dynamical Systems, and Bifurcation of Vector Fields (Springer, New York, 1983).

Hart-Smith, L. J., “Elasticity parameters for finite deformations of rubber-like materials,' Z. Angew. Math. Phys. 17, 608-626 (1966).

Isihara, A., N. Hashitsume, and M. Tatibana, "Statistical theory of rubber-like elasticity_IV,' J. Chem. Phys. 19, 1508-1512 (1951).

Jenkins, C. H. and J. W. Leonard, “Nonlinear dynamic response of membranes: State of the art,' Appl. Mech. Rev. 44, 319-328 (1991).

Jenkins, C. H., 'Nonlinear dynamic response of membranes: State of the art—Update,' Appl. Mech. Rev. 49, S41-S48 (1996).

Khayat, R. E. and A. Garcia-Rejon, "Uniaxial and biaxial unsteady inflations of a viscoelastic material,' J. Non-Newtonian Fluid Mech. 43, 31-59 (1992).

Khayat, R. E., A. Derdouri, and A. Garcia-Rejon, "Inflation of an elastic cylindrical membrane: Non-linear deformation and instability," Int. J. Solids Struct. 29, 69-87 (1992).

Khayat, R. E., A. Derdouri, and A. Garcia-Rejon, "Multiple contact and axisymmetric inflation of a hyperelastic cylindrical membrane,' J. Mech. Eng. Sci. 207, 175-183 (1993).

Khayat, R. E. and A. Derdouri, "Inflation of hyperelastic cylindrical membranes as applied to blow moulding. Part I. Axisymmetric Case,’ Int. J. Numer. Methods Eng. 37, 3773-3792 (1994a).

Khayat, R. E. and A. Derdouri, "Inflation of hyperelastic cylindrical membranes as applied to blow moulding. Part II. Non-axisymmetric case,’ Int. J. Numer. Methods Eng. 37, 3793-3808 (1994b).

Khayat, R. E. and A. Derdouri, "Stretch and inflation of hyperelastic membranes as applied to blow molding," Polym. Eng. Sci. 35, 1852-1863 (1995).

Khayat, R. E. and P. Raducanu, "A coupled finite element/boundary element approach for the threedimensional simulation of air venting in blow molding and thermoforming,' Int. J. Numer. Methods Eng. 43, 151-174 (1998).

Mooney, M., “A theory of large elastic deformation,” J. Appl. Phys. 11, 582-592 (1940).

Ogden, R. W., "Large deformation isotropic elasticity—on the correlation of theory and experiment for incompressible rubberlike solids," Proc. R. Soc. London, Ser. A 326, 565-584 (1972).

Rivlin, R. S., "Large elastici deformation of isotropic materials-IV. Further developments of the general theory," Philos. Trans. R. Soc. London, Ser. A 241, 379-397 (1948).

Rivlin, R. S. and D. W. Saunders, "Large elastici deformation of isotropic materials-VII. Experiments on the deformation of rubber," Philos. Trans. R. Soc. London, Ser. A 243, 251-288 (1951).

Shang, X. C., "Tensile instability of nonlinear spherical membrane with large deformation," Appl. Math. Mech. (in Chinese) 12, 993-1000 (1991).

Treloar, L. R., The Physics of Rubber Elasticity, 3rd ed. (Clarendon, Oxford, 1975).

Valenis, K. C. and R. F. Landel, "The stain-energy function of a hyperelastic material in terms of the extension ratios,'’ J. Appl. Phys. 38, 2997-3002 (1967). 